\section{RMD Open}

Rheumatic \&

Musculoskeletal Diseases

\title{
Switching biological disease-modifying antirheumatic drugs in patients with axial spondyloarthritis: results from a systematic literature review
}

\author{
Victoria Navarro-Compán, ${ }^{1}$ Chamaida Plasencia-Rodríguez, ${ }^{1}$ Eugenio de Miguel, ${ }^{1}$ \\ Petra Diaz del Campo, ${ }^{2}$ Alejandro Balsa, ${ }^{1}$ Jordi Gratacós ${ }^{3}$
}

To cite: Navarro-Compán V, Plasencia-Rodríguez C, de Miguel E, et al. Switching biological disease-modifying antirheumatic drugs in patients with axial spondyloarthritis: results from a systematic literature review. RMD Open 2017;3:e000524. doi:10.1136/ rmdopen-2017-000524

- Prepublication history and additional material for this paper are available online. To view please visit the journal (http://dx doi.org/10.1136/rmdopen-2017000524).

Received 21 June 2017 Revised 30 August 2017 Accepted 15 September 2017

\section{(a) CrossMark}

${ }^{1}$ Department of Rheumatology, University Hospital La Paz, IdiPAZ, Madrid, Spain

${ }^{2}$ Research Unit, Spanish Society of Rheumatology, Madrid, Spain ${ }^{3}$ Department of Rheumatology, University Hospital Parc Taulí (ISPT), UAB, Sabadell, Barcelona Spain

Correspondence to Dr Victoria Navarro-Compán; mvictoria.navarroc@gmail.com

\section{ABSTRACT}

Objectives First, to investigate if switching biological disease-modifying antirheumatic drugs (bDMARDs) after the failure to prior bDMARD is efficacious in patients with axial spondyloarthritis (axSpA). Second, to evaluate the influence on this efficacy of (1) the reason to discontinue prior tumour necrosis factor inhibitor (TNFi), (2) changing the type of TNFi and (3) changing the target.

Methods A systematic literature review until January 2017 was performed using Medline, EMBASE and Cochrane databases. Longitudinal studies assessing clinical response after switching bDMARDs in patients with axSpA were analysed

Results In total, 9 studies out of 1862 retrieved citations were included. Overall, the level of evidence was poor. In these studies, all patients received a TNFi as first bDMARD, 1956 patients switched to a second bDMARD (97\% TNFi and $3 \%$ interleukin-17 inhibitors (IL-17i)) and 170 to a third bDMARD (all TNFi). Clinical response (Bath Ankylosing Spondylitis Disease Activity Index 50) after a second TNFi was achieved by $25 \%-56 \%$ of patients compared with $50 \%-72 \%$ after the first TNFi. Also, $47 \%$ of patients switching to IL-17i after a TNFi responded (Assessment of SpondyloArthritis international Society 40) compared with $66 \%$ in those who received IL-17i as first line. The response after switching was not influenced by the reason to discontinue, type of prior TNFi or changing the target. Conclusions In patients with axSpA, switching to a second bDMARD (a TNFi or IL-17i) after prior TNFi is efficacious. Nevertheless, the clinical response is lower than the observed in patients naive to bDMARD. So far, the reason to discontinue prior bDMARD or the type of bDMARD has not been identified as predictor of response. Published evidence for switching to a third bDMARD is lacking.

\section{INTRODUCTION}

Biological disease-modifying antirheumatic drugs (bDMARDs) have shown to be efficacious for improving clinical symptoms and signs in patients with axial spondyloarthritis $(\operatorname{axSpA}) .^{1-6}$ Regarding this therapy, only tumour necrosis factor inhibitors (TNFi) were indicated in the past, but recently

\section{Key messages}

What is already known about this subject?

- In patients with axial spondyloarthritis, the evidence to support switching to a second or consecutive biological disease-modifying antirheumatic drug (bDMARD) is unclear.

What does this study add?

- The results of this systematic literature review support that switching to a second bDMARD (a tumour necrosis factor inhibitor (TNFi) or interleukin-17 inhibitor) after the failure to prior TNFi is efficacious.

- Nevertheless, the clinical response after the second bDMARD is lower than that observed in patients naive to bDMARD.

How might this impact on clinical practice?

- Switching to a second bDMARD after the failure to prior TNFi is a reasonable practice supported by scientific evidence.

interleukin-17 inhibitors (IL-17i) have also been approved-so far, in patients with ankylosing spondylitis (AS). Unfortunately, clinical response after a bDMARD is not achieved by all patients with axSpA and a significant proportion of them had failure to this therapy. Data from clinical trials show that 50\%-65\% of patients with axSpA do not achieve clinical response (defined as Assessment of SpondyloArthritis international Society (ASAS) 40) after receiving a TNFi during 24 weeks as firstline bDMARD therapy, ${ }^{2-6}$ and similar results have also been observed with IL-17i, with $58 \%-64 \%$ of patients not reaching ASAS40 response after 16 weeks of treatment. ${ }^{1}$ In clinical practice, data are available only for TNFi, which indicate that after 2 years of treatment up to $45 \%$ of patients already interrupt this therapy. $^{78}$ 
If a first bDMARD is not efficacious, a common clinical practice is switching to another bDMARD. ${ }^{9-11}$ In fact, this attitude is so extended and accepted that it has been included in the recent update of the ASAS/European League Against Rheumatism management recommendations for patients with axSpA. ${ }^{12}$ However, the evidence to support switching to a second or consecutive bDMARD in these patients is unclear. In this regard, several limitations in the field of axSpA make it difficult to summarise the published literature on this topic. ${ }^{13}$ First, the definition of failure in axSpA is not well established. Usually, this is defined as either intolerance or adverse events related to the drug or inefficacy to this, which can be distinguished between primary (non-response) or secondary (loss of response) failure. ${ }^{14}$ Nevertheless, other scenarios such as radiographical progression or persistence of inflammatory lesions on MRI could also be used to define a failure in patients with axSpA. Second, the definition of clinical response employed in the studies including patients with axSpA is very heterogeneous and depends on the context. In clinical trials, response is commonly defined as achieving the ASAS response criteria (ASAS20 or ASAS40), while observational studies usually employ a more simple but less strict definition such as the response based on the Bath Ankylosing Spondylitis Disease Activity Index (BASDAI50 or a decrease of at least 2 units in a scale of 0-10) or the Ankylosing Spondylitis Disease Activity Score (ASDAS) with a decrease of at least 1.1 units (for clinical improvement) or 2 units (for major improvement). ${ }^{15} 16$ Furthermore, there is no valid definition to establish what is a flare in patients with axSpA. Recently, 12 possible scenarios have been proposed, but a final definition has not been validated yet. ${ }^{17}$ Finally, when analysing and interpreting data from scientific literature to support the common current practice of switching bDMARDs in patients with axSpA, it also needed to be considered that several factors may influence the response to bDMARDs in these patients, including characteristics of the patients (gender, age, smoking habit, human leukocyte antigen-B27 (HLAB27) carriage and body mass index) or characteristics of the disease (duration and activity degree) at the beginning of the treatment. ${ }^{18}$

Additionally, it also remains unanswered whether or not the reason to discontinue a bDMARD influences the response to the next administered bDMARD in patients with axSpA. Also, the influence of changing the type of TNFi (from monoclonal antibody to receptor fusion protein or vice versa) or the target mechanism (TNF to IL-17 or vice versa) on the response to consecutive bDMARDs is unclear. Until last year, switching from a TNFi to another TNFi was the only option, but now other possibilities are available, which makes it even more necessary to answer all these pending questions in order to provide some guidance to all clinicians for selecting the most appropriate approach in patients with axSpA interrupting a first bDMARD.
Based on this, the main objective of this study was to determine if switching to another bDMARD after the failure to a bDMARD is efficacious in patients with axSpA. The second objective is to evaluate the influence of (1) the reason to discontinue the bDMARD, and to analyse (2) the change of type of TNFi and (3) the change of target mechanism.

\section{METHODS}

Research clinical question and search strategy

A systematic literature review was performed using Medline, EMBASE and Cochrane databases in collaboration with an epidemiologist with expertise on SLR methodology (PDdC). The research clinical question was formulated according to the PICOS (Population, Intervention, Comparison, Outcome and Study design) method. The definition for each of these items was defined as follows:

- population: patients with axSpA including AS and non-radiographical axSpA (nr-axSpA) who experienced failure to at least one bDMARD; for this purpose, there was no pre-established definition of failure, but the reason to interrupt treatment was collected and reported for each study

- intervention: second or consecutive bDMARD

- outcome: clinical response, indicated by at least one of the following response criteria: ASAS (ASAS20 or ASAS40), BASDAI (BASDAI50 or $\triangle$ BASDAI $\geq 2$ units) and ASDAS (clinically important improvement defined as ASDAS $\Delta \geq 1.1$ units or major improvement defined as ASDAS $\Delta \geq 2.0$ units) $)^{1516}$

- setting: longitudinal studies with at least 12 weeks of follow-up, including at least 50 patients with the characteristics defined in the population.

The search did not have any limit for starting date and included published studies up to 1 January 2017. Studies published in English, Spanish or French were included. The search terms are provided in online supplementary file table $\mathrm{S} 1$.

\section{Selection of studies}

First, titles and abstracts of the citations were screened. Later, after reading the full text of the preselected articles, it was decided whether or not to include a study for data extraction. Inclusion criteria were observational or interventional studies evaluating the efficacy of a second or consecutive bDMARD in patients with axSpA who have experienced a failure to a first bDMARD. Exclusion criteria were not in compliance with the definitions established for the items on the PICOs or insufficient data to evaluate the objective of the study.

\section{Data extraction and data summary}

Data were extracted using a specific form developed for this purpose. Two readers (VN-C and CP-R) independently extracted data for each study, including characteristics of the studies (year of publication, journal, design, sample size, follow-up period), characteristics 


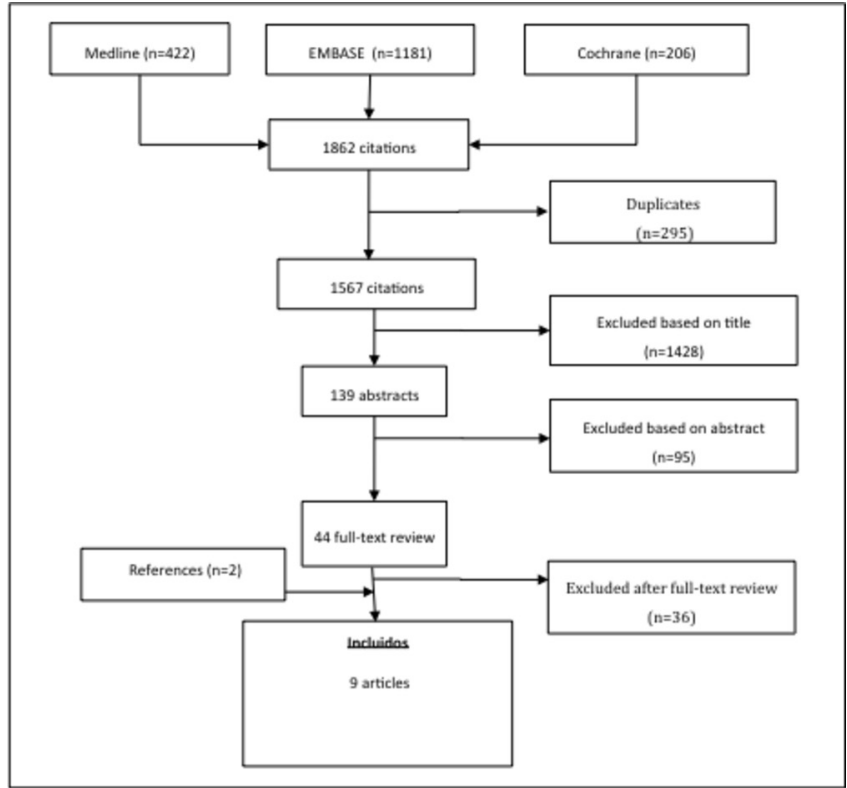

Figure 1 Algorithm for the research strategy and selection of the studies included in the systematic literature review.

of patients included in each study (demographic and disease characteristics), treatment received as first or consecutive bDMARD and reason to interrupt the treatment and outcome (measure employed to define clinical response and percentage of patients who achieved this). Further, the quality and potential biases of the studies assigning an overall quality score per study between 0 and 5 points according Oxford 2009 level of evidence were also evaluated.

\section{RESULTS}

\section{Characteristics of the studies}

A detailed flow chart with the results of the literature search is depicted in figure 1 .

The search retrieved 1862 citations. A total of 44 articles were selected for full-text review, out of which nine studies were finally included for data extraction. ${ }^{9-11} 14$ 19-23 The reasons to exclude the remaining studies after reading the full text are provided in online supplementary file table S2). Details for each of the nine studies included are shown in table 1.

Most of them were observational studies reporting data from national registries and only two studies used data from clinical trials. In total the selected studies included data from 1956 patients (90.9\% AS and 9.1\% nr-axSpA) who switched to a second bDMARD and 170 patients (all of them with AS) who switched to a third bDMARD. Importantly, the first bDMARD was a TNFi in all patients, while the second bDMARD was a TNFi in 1905 (97.4\%) patients and an IL-17i in 51 (2.6\%) patients. Baseline characteristics (median, range) of patients who switched bDMARD were as follows: age 43 (38-46) years old, $67 \%$ (54-80) male, 77\% (62-84) HLA-B27+ and BASDAI before switching drugs 6.2 (5.2-7.1). As a control group, data from 4191 patients after receiving the first bDMARD were analysed. Median (range) follow-up period after switching bDMARD was 6 (3-12) months. The level of evidence according to Oxford 2009 was 4 for seven studies and $2 \mathrm{~b}$ for the remaining two studies.

\section{Reasons to discontinue the first bDMARD}

Figure 2 shows the reasons to discontinue the first bDMARD for the six studies reporting data on this. 7114192022 The most common reason was inefficacy, followed by intolerance or adverse events related to the administered drug. With regard to the type of inefficacy, only three studies differentiated between primary and secondary failure. ${ }^{14}{ }^{192}$ In these studies, primary failure was the reason to interrupt bDMARD in a smaller subgroup of patients $(19 \%, 22 \%$ and $23 \%$, respectively), while secondary failure was the responsible cause in a higher proportion of patients $(35 \%, 39 \%$ and $43 \%$, respectively).

\section{Efficacy of switching to a second bDMARD}

In total, nine studies evaluated the efficacy of switching to a second bDMARD. All patients participating in these received a TNFi as the first bDMARD. Additionally, the second bDMARD was a TNFi in eight out of nine studies including 1905 patients, while this was an IL-17i in only one study reporting pooled data from a total of 51 patients participating in Efficacy, Safety and Tolerability of Secukinumab in Active Ankylosing Spondylitis Patients (MEASURE) 1 and MEASURE 2 trials. The type of TNFi administered as first or second bDMARD is specified in table 1.

Figure 3 shows the percentage of patients achieving clinical response for each study. Importantly, the sample size and the definition of clinical response employed were very heterogeneous across the studies. The number of patients switching to a second TNFi ranged between 77 and 632. The percentage of patients achieving clinical response after a second TNFi ranged between $25 \%$ and $56 \%$ in six studies using BASDAI50, $78 \%$ in one study using BASDAI $<4 \%$ and $17 \%$ in one study using ASASpr criteria, which is a more strict definition. In these same studies, clinical response after the first TNFi occurred in $50 \%-72 \%$ and $83 \%$ of patients, respectively (no data reported for the study using ASASpr criteria). Furthermore, $47 \%$ of 51 patients receiving IL-17i after a prior TNFi achieved clinical response (ASAS40 criteria) compared with $66 \%$ of patients who received IL-17i as the first bDMARD.

\section{Efficacy of switching to a third bDMARD}

Only three studies including a total of 170 patients evaluated the efficacy of a third bDMARD, all of them after receiving two TNFis, which are also specified in table 1. Clinical response was defined as BASDAI50 in the three studies, and the percentage of patients achieving this outcome was $29 \%,{ }^{19} 30 \%{ }^{9}$ and $52 \% .^{20}$

No study provided data on the efficacy of a fourth or consecutive bDMARD in patients with axSpA. 


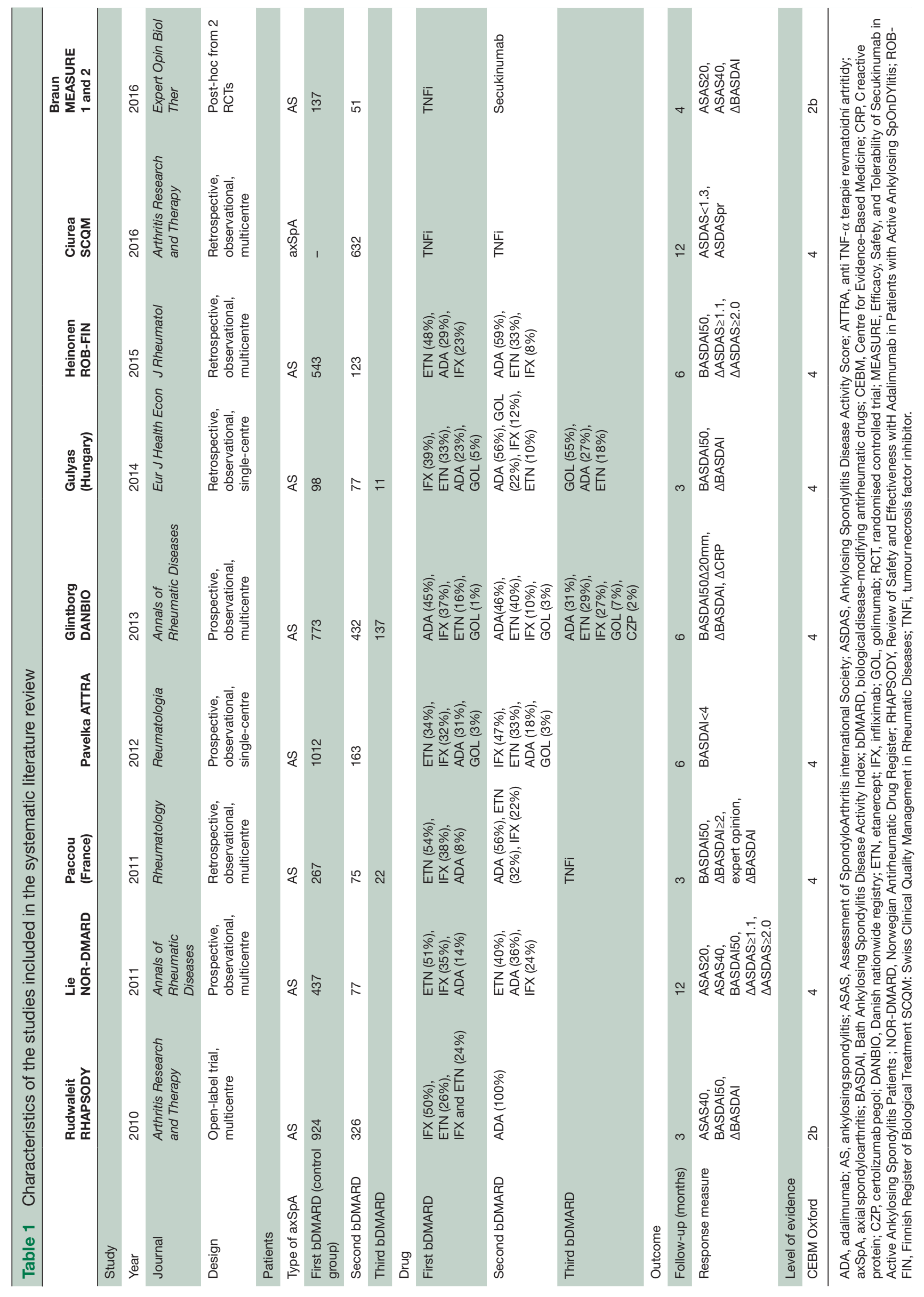




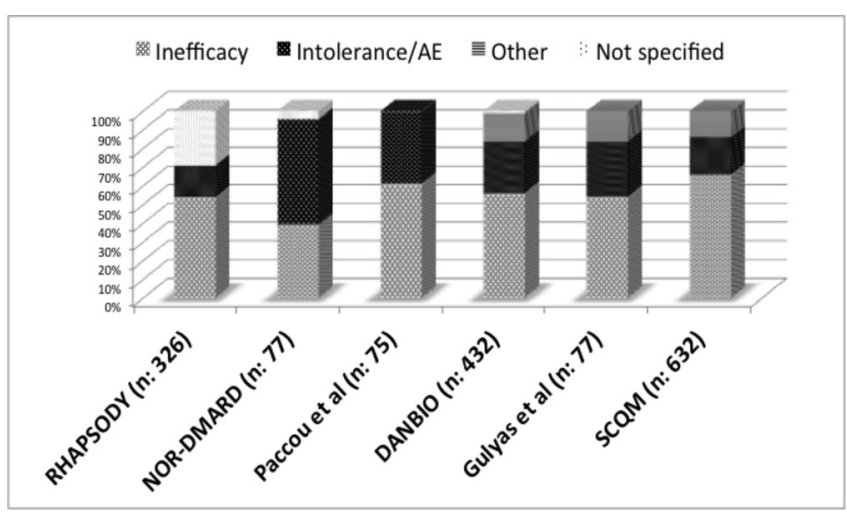

Figure 2 Reasons to discontinue the first biological disease-modifying antirheumatic drug (antitumour necrosis factor in all studies). AE, adverse event.

\section{Predictors of clinical response after switching bDMARDs Reason to discontinue the first bDMARD}

In total, five studies evaluated the influence of the reason to interrupt the first bDMARD (a TNFi in all studies) on the efficacy of the second or third bDMARD (also a TNFi in all studies).

Two of these studies differentiated between inefficacy and adverse events, but they did not provide data about the type of failure (primary or secondary). In the Norwegian Antirheumatic Drug Register (NOR-DMARD study $(n=77)$, the percentage of patients achieving BASDAI50 response was similar in patients who discontinued drug due to inefficacy compared with that observed in patients interrupting it because of adverse events (21\% vs $33 \%$, respectively; $\mathrm{p}=0.36) .{ }^{11}$ In the Danish nationwide registry (DANBIO) registry $(\mathrm{n}=432)$, the reason to discontinue the first TNFi was not significantly predictive of response to the second TNFi, and drug survivals were similar among groups regardless of the reason for switching. ${ }^{9}$

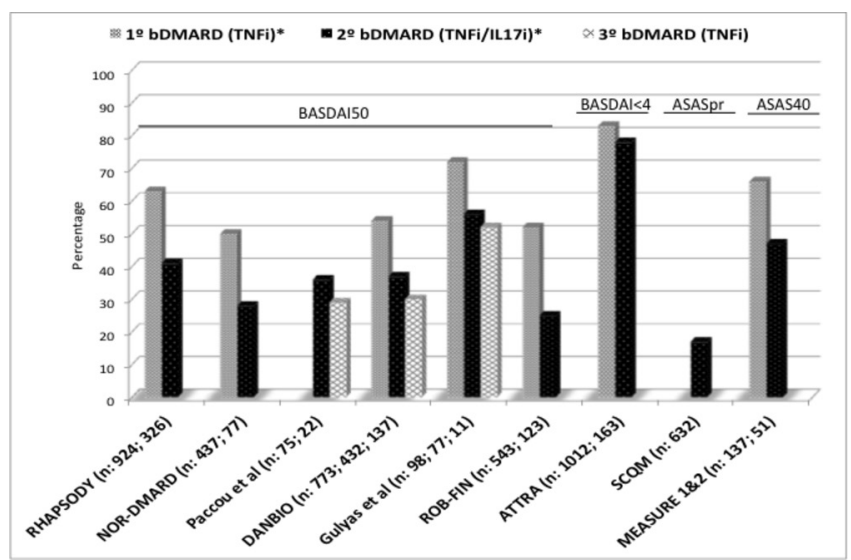

Figure 3 Efficacy as first, second and third bDMARD in patients with axial spondyloarthritis. ASAS, Assessment of Spondylo Arthritis international Society; BASDAI, Bath Ankylosing Spondylitis Disease Activity Index; bDMARD, biological disease-modifying antirheumatic drugs; IL-17i, interleukin-17 inhibitor; TNFi, tumour necrosis factor inhibitor.
Additionally, three studies also differentiated between primary or secondary failure within patients who discontinued because of inefficacy. The RHAPSODY study $(\mathrm{n}=326)$ demonstrated in a logistic regression analysis that the reason to discontinue the prior TNFi had no statistically important impact on the likelihood to achieve clinical response. Nevertheless, the observed ASAS40 and BASDAI50 response rates were greater in the group of patients discontinuing prior TNFi therapy because of loss of response or intolerance than in the group interrupting the first TNFi due to lack of response (ASAS40 response: $43 \%$ and $39 \%$ vs $26 \%$, respectively; BASDAI50 response: $42 \%$ and $46 \%$ vs $26 \%$, respectively). ${ }^{14}$ In the study from Paccou et al $(\mathrm{n}=75)$, the response to the second or third TNFi was not influenced by the reason to interrupt the first TNFi either. In this, the percentage of responders to a second TNFi was $79 \%$ for side effect, $82 \%$ for loss of efficacy and $81 \%$ for primary non-responders. ${ }^{19}$ Opposite this, Ciurea et al $(\mathrm{n}=632)$ recently reported in a Swiss cohort that the efficacy of a second TNFi is significantly impaired in patients with primary failure compared with those with secondary failure. The median drug survival was lower for primary versus secondary failure (1.1 vs 3.8 years, respectively; $\mathrm{p}<0.01$ ), and the percentage of patients achieving at least a moderate disease activity according to the ASDAS was also lower in the first group ( $11 \%$ vs $39 \%$, respectively; $\mathrm{p}<0.01$ ). Nevertheless, the proportion of HLA-B27 carriers within the subgroup of patients experiencing primary failure was significantly lower than among patients with secondary failure (43\% vs $69 \%$, respectively; $\mathrm{p}<0.001$ ), which could also explain the differences observed in clinical response after switching to a second TNFi, because HLA-B27 has been associated with clinical response to TNFi and because this could represent misdiagnosis of axSpA among the primary failure subgroup. ${ }^{24}$

\section{Changing the type of TNFi}

Only the RHAPSODY study analysed if the probability to achieve clinical response after switching depended on the type of prior TNFi received. In this open-label study, patients who experienced a failure to etanercept $(n=85)$, infliximab $(n=150)$ or both TNFis $(n=74)$ received adalimumab. Surprisingly, results showed that the likelihood of achieving ASAS40 response after 12 weeks of adalimumab was significantly greater for patients with only prior infliximab therapy compared with patients with only prior etanercept therapy and those with prior treatment with both infliximab and etanercept (44\% vs $31 \%$ and $32 \%$, respectively). ${ }^{14}$

\section{Changing the target mechanism}

Data from switching to a different target only come from a pooled analysis using data of the MEASURE 1 and MEASURE 2 trials. In these studies, a total of 51 patients switched from TNFi to IL-17i, but the reason to discontinue TNFi was not reported in detail. Out of these patients, $47 \%$ achieved clinical response (ASAS40 
criteria) after 16 weeks of treatment. ${ }^{23}$ Finally, so far there are no data available to assess the efficacy of receiving a TNFi after being treated previously with IL-17i.

\section{DISCUSSION}

This study summarises the scientific evidence to switch bDMARDs in patients with axSpA. In addition, it also analyses the influence of three relevant factors (reason to discontinue prior bDMARD, changing the type of TNFi received and changing the target mechanism) on the probability to achieve clinical response after switching to a second or consecutive bDMARD in these patients.

Published data indicate that switching to a second bDMARD (either a TNFi or IL-17i) in patients with axSpA interrupting a prior TNFi is efficacious. However, clinical response after this is lower than the one experienced by patients naive to bDMARD. Between 25\% and $56 \%$ of patients switching to a second TNFi achieve clinical response (BASDAI50), which is similar to the ASAS40 response observed data in patients who switch to an IL-17i (30\%-50\%). Moreover, published data to assess the efficacy of switching to a third bDMARD (only TNFi data are available) are very limited and do not allow making strong conclusions. However, it seems that the likelihood to response after a second switch is lower than after the first switch.

In addition, this review also analyses the influence of three important factors as possible predictors of clinical response when switching bDMARD in patients with axSpA: (1) the reason to discontinue prior TNFi, (2) changing the type of TNFi received and (3) changing the target mechanism. Opposite to the results observed in patients with rheumatoid arthritis ${ }^{25}$ no predictors have been clearly identified, but it is also true that published data to answer this question in patients with axSpA are very limited, especially to evaluate the influence of changing the type of TNFi received or the target mechanism.

To our knowledge, this is the first study systematically reviewing the efficacy of switching bDMARD in patients with axSpA. Nevertheless, a few relevant limitations should be taken into consideration. Overall, it is difficult to compare results among studies because the sample size, design, context and clinical measures employed to assess clinical response are very heterogeneous. For switching to a second TNFi, extensive data from almost 2000 patients are available, but most of these come from observational studies using BASDAI50 response criteria as the outcome. Moreover, in these studies, not all patients discontinuing a first TNFi switched to a second TNFi, which may overestimate the response rate among these patients. ${ }^{9}$ By contrary, available data for switching to an IL-17i are just limited to 51 patients, but all of them participating in two randomised controlled trials using ASAS40 criteria to define clinical response. Therefore, the comparison between drugs should be done with caution. In addition, this systematic literature review assessed efficacy of bDMARDs based on clinical response measures, but it did not analyse drug survival in the different studies, which may affect the results. In this regard, it was preferred to use a more objective outcome. Furthermore, most of the studies included patients with $\mathrm{AS}$, so the evidence in patients with nr-axSpA is almost non-existent.

In conclusion, in patients with axSpA, published data indicate that switching to a second bDMARD (either a TNFi or IL-17i) after interrupting a prior TNFi is efficacious. However, clinical response after this second bDMARD is lower than the one achieved by patients naive to bDMARD. So far, considering the reason to discontinue the first bDMARD, the type of prior TNFi or the change of target mechanism does not seem to influence on the response to the second bDMARD. Nevertheless, with regard to changing target data are very limited and restricted to one direction (from TNFi to IL-17i). Finally, data to assess the efficacy of switching to a third bDMARD in patients with axSpA are lacking and not conclusive, so further data are necessary to answer this question. On top of this, future studies should include patients covering the entire spectrum of the disease and a headto-head comparison between the different alternatives to switch bDMARDs.

Acknowledgements This SLR was performed as part of the development process of the Recommendations for the Use of Biological Therapies in Axial Spondyloarthritis of the Spanish Society of Rheumatology. The authors also thank Mercedes Guerra and María García-Puente for their assistance in performing the search.

Contributors VN-C wrote the first draft of the manuscript. VN-C and CPR extracted and summarised the data. PDdC, AB, EDM and JG critically reviewed the manuscript. All authors approved the final version of the manuscript.

Funding VN-C received funding from the Spanish Society of Rheumatology to perform the SLR.

Competing interests VN-C has received speaking fees or funding for research projects and attending congresses from AbbVie, BMS, Lilly, MSD, Novartis, Pfizer, Roche and UCB. AB is a consultant for, participated in clinical trials for, member of a speaker bureau for, received research grants from Abbvie, Boehringer, BMS, MSD, Novartis, Pfizer, UCB, MSD and Roche. CPR has received grants from Pfizer. EDM has been a consultant for, participated in clinical trials for, member of a speaker bureau for and received research grants from AbbVie, Amgen, Biogen, BMS, Janssen, Hospira, MSD, Novartis, Pfizer, Roche, Sanofi and UCB. JG is a consultant for, participated in clinical trials for, member of a speaker bureau for, received research grants from AbbVie, MSD, Novartis, Pfizer, UCB and Janssen. The other author has declared no conflicts of interest.

Provenance and peer review Not commissioned; externally peer reviewed.

Open Access This is an Open Access article distributed in accordance with the Creative Commons Attribution Non Commercial (CC BY-NC 4.0) license, which permits others to distribute, remix, adapt, build upon this work non-commercially, and license their derivative works on different terms, provided the original work is properly cited and the use is non-commercial. See: http://creativecommons.org/ licenses/by-nc/4.0/

(C) Article author(s) (or their employer(s) unless otherwise stated in the text of the article) 2017. All rights reserved. No commercial use is permitted unless otherwise expressly granted.

\section{REFERENCES}

1. Baeten D, Sieper J, Braun J, et al. Secukinumab, an interleukin-17A inhibitor, in ankylosing spondylitis. N Engl J Med 2015;373:2534-48.

2. Davis JC, van der Heijde DM, Braun J, et al. Sustained durability and tolerability of etanercept in ankylosing spondylitis for 96 weeks. Ann Rheum Dis 2005;64:1557-62. 
3. Inman RD, Davis JC, Heijde D, et al. Efficacy and safety of golimumab in patients with ankylosing spondylitis: results of a randomized, double-blind, placebo-controlled, phase III trial. Arthritis Rheum 2008;58:3402-12.

4. Landewé R, Braun J, Deodhar A, et al. Efficacy of certolizumab pegol on signs and symptoms of axial spondyloarthritis including ankylosing spondylitis: 24-week results of a double-blind randomised placebo-controlled phase 3 study. Ann Rheum Dis 2014;73:39-47.

5. van der Heijde D, Dijkmans B, Geusens P, et al. Efficacy and safety of infliximab in patients with ankylosing spondylitis: results of a randomized, placebo-controlled trial (ASSERT). Arthritis Rheum 2005;52:582-91.

6. van der Heijde D, Kivitz A, Schiff MH, et al. Efficacy and safety of adalimumab in patients with ankylosing spondylitis: results of a multicenter, randomized, double-blind, placebo-controlled trial. Arthritis Rheum 2006;54:2136-46.

7. Glintborg B, Ostergaard M, Krogh NS, et al. Predictors of treatment response and drug continuation in 842 patients with ankylosing spondylitis treated with anti-tumour necrosis factor: results from 8 years' surveillance in the Danish nationwide DANBIO registry. Ann Rheum Dis 2010;69:2002-8.

8. Moral E, Plasencia C, Navarro-Compán V, et al. AB0657 Discontinuation of anti-tnf therapy in patients with axial spondyloarthritis in clinical practice: prevalence and causes. Ann Rheum Dis 2016;75:1129.1-1129.

9. Glintborg B, Østergaard M, Krogh NS, et al. Clinical response, drug survival and predictors thereof in 432 ankylosing spondylitis patients after switching tumour necrosis factor $\alpha$ inhibitor therapy: results from the Danish nationwide DANBIO registry. Ann Rheum Dis 2013:72:1149-55

10. Pavelka K, Fojtíková M, Hejduk K. Efficacy of the first and subsequent courses of anti-TNF therapy in patients with ankylosing spondylitis - results from the Czech National Register ATTRA. Reumatologia 2012;50:294-306.

11. Lie $\mathrm{E}$, van der Heijde D, Uhlig T, et al. Effectiveness of switching between TNF inhibitors in ankylosing spondylitis: data from the NOR-DMARD register. Ann Rheum Dis 2011;70:157-63.

12. van der Heijde D, Ramiro S, Landewé R, et al. 2016 update of the ASAS-EULAR management recommendations for axia spondyloarthritis. Ann Rheum Dis 2017;76:978-91.

13. Deodhar A, Yu D. Switching tumor necrosis factor inhibitors in the treatment of axial spondyloarthritis. Semin Arthritis Rheum 2017.
14. Rudwaleit M, Van den Bosch F, Kron M, et al. Effectiveness and safety of adalimumab in patients with ankylosing spondylitis or psoriatic arthritis and history of anti-tumor necrosis factor therapy. Arthritis Res Ther 2010;12:R117.

15. Machado P, Landewé R, Lie E, et al. Ankylosing Spondylitis Disease Activity Score (ASDAS): defining cut-off values for disease activity states and improvement scores. Ann Rheum Dis 2011;70:47-53.

16. Sieper J, Rudwaleit M, Baraliakos X, et al. The Assessment of SpondyloArthritis international Society (ASAS) handbook: a guide to assess spondyloarthritis. Ann Rheum Dis 2009;68(Suppl 2):ii1-44

17. Gossec L, Portier A, Landewé R, et al. Preliminary definitions of 'flare' in axial spondyloarthritis, based on pain, BASDAI and ASDASCRP: an ASAS initiative. Ann Rheum Dis 2016;75:991-6.

18. Maneiro JR, Souto A, Salgado E, et al. Predictors of response to TNF antagonists in patients with ankylosing spondylitis and psoriatic arthritis: systematic review and meta-analysis. RMD Open 2015;1:e000017.

19. Paccou J, Solau-Gervais E, Houvenagel E, et al. Efficacy in current practice of switching between anti-tumour necrosis factor- $\alpha$ agents in spondyloarthropathies. Rheumatology 2011;50:714-20.

20. Gulyas K, Bodnar N, Nagy Z, et al. Real-life experience with switching TNF- $\alpha$ inhibitors in ankylosing spondylitis. Eur J Health Econ 2014;15(Suppl 1):93-100

21. Heinonen AV, Aaltonen KJ, Joensuu JT, et al. Effectiveness and drug survival of TNF inhibitors in the treatment of Ankylosing Spondylitis: a prospective cohort study. J Rheumatol 2015;42:2339-46.

22. Ciurea $A$, Exer $P$, Weber $U$, et al. Does the reason for discontinuation of a first TNF inhibitor influence the effectiveness of a second TNF inhibitor in axial spondyloarthritis? results from the Swiss clinical quality management cohort. Arthritis Res Ther 2016;18:71.

23. Braun J, Baraliakos X, Kiltz U. Secukinumab (AIN457) in the treatment of ankylosing spondylitis. Expert Opin Biol Ther 2016;16:711-22.

24. Baraliakos X, Borah B, Braun J, et al. Long-term effects of secukinumab on MRI findings in relation to clinical efficacy in subjects with active ankylosing spondylitis: an observational study. Ann Rheum Dis 2016;75:408-12.

25. Cantini F, Niccoli L, Nannini C, et al. Second-line biologic therapy optimization in rheumatoid arthritis, psoriatic arthritis, and ankylosing spondylitis. Semin Arthritis Rheum 2017 (Epub ahead of print: 22 Mar 2017). 